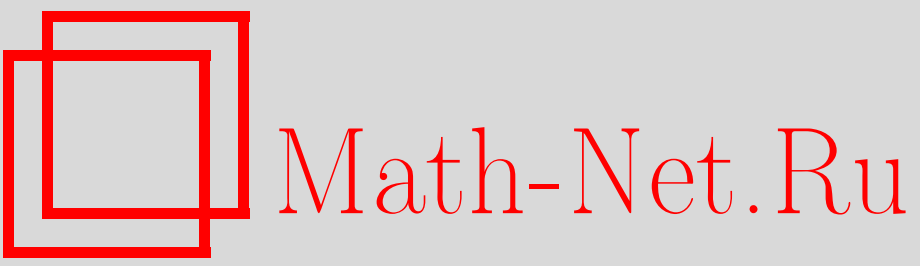

А. Я. Казаков, Интегральные симметрии, интегральные инварианты и матрицы монодромии для обыкновенных дифференциальных уравнений, ТМФ, 1998, том 116, номер 3, 323-335

DOI: https://doi.org/10.4213/tmf906

Использование Общероссийского математического портала Math-Net.Ru подразумевает, что вы прочитали и согласны с пользовательским соглашением

http://www.mathnet.ru/rus/agreement

Параметры загрузки:

IP : 54.237.59.107

26 апреля 2023 г., 12:46:35 
ТЕОРЕТИЧЕСКАЯ

И МАТЕМАТИЧЕСКАЯ

ФИЗИКА

Том 116, № 3

сентябрь, 1998

(C) 1998 г.

А.Я. Казаков*

\section{ИНТЕГРАЛЬНЫЕ СИММЕТРИИ, ИНТЕГРАЛЬНЫЕ ИНВАРИАНТЫ И МАТРИЦЫ МОНОДРОМИИ ДЛЯ ОБЫКНОВЕННЫХ ДИФФЕРЕНЦИАЛЬНЫХ УРАВНЕНИЙ}

Рассматриваются матрицы связи и монодромии для вырожденного уравнения Гойна. Используется вспомогательное обыкновенное линейное дифференциальное уравнение третьего порядка. Данное уравнение "устойчиво" относительно интегрального преобразования Эйлера. Найден инвариант этого преобразования, который выражается через элемент матрицы связи.

\section{1. ВВЕДЕНИЕ}

Классические специальные функции (решения гипергеометрического уравнения и его вырожденных случаев) находят широкое применение в различных задачах прикладной математики и физики. Эти функции давно и успешно изучаются, обширная информация об их свойствах изложена в доступных руководствах [1-3]. Наличие интегральных представлений для решений гипергеометрического уравнения дает, в частности, возможность в явном виде построить группу матрищ монодромии для гипергеометрического уравнения и для его редукций. Однако для решений более сложных уравнений, например для уравнения Гойна [4] и его различных вырожденных случаев, интегральных представлений нет. В связи с этим необходимо развивать иные способы получения аналитической информации о решениях подобных уравнений. Определенные возможности в этом смысле дают интегральные соотношения, которые можно получить для широкого класса линейных обыкновенных дифференциальных уравнений [5-11]. Так, в работе [10] интегральные соотношения использовались при выводе интегральных уравнений типа $\Phi$ редгольма для решений спектральных задач, связанных с уравнениями класса Гойна, а в [11] - для описания соотношений между решениями спектральных задач. Двойственность между различными дифференциальными уравнениями (которая выражается с помощью интегральной связи) была использована в работе [12] при построения численной процедуры вычисления спектральных параметров соответствуюших дифферен-

* Государственный университет аэрокосмического приборостроения, С.-Петербург, Россия. E-mail: akaz@phsc2.neva.ru 
циальных операторов. В данной работе мы используем интегральные соотношения для изучения матриц связи соответствуюших дифференциальных уравнений.

Обсуждаемая в данной работе проблема примыкает к известной в математике проблеме Римана-Гильберта (21-й проблеме Гильберта [13, 14]): существует ли для известной группы матриц обыкновенное линейное дифференциальное уравнение, для которого данная группа является группой матрищ монодромии. Для практических приложений более важна обратная задача - задача построения по данному уравнению группы матриц монодромии. Как уже упоминалось выше, полная программа в этом направлении осуществлена только для гипергеометрического уравнения на основе интегральных представлений его решений. Эти результаты были получены в середине XIX века Куммером и Риманом (см. [1]). Для более сложных уравнений никакого продвижения с тех пор не было.

В данной работе предлагается способ аналитического вычисления матриц связи (через которые можно выразить и матрицы монодромии) для уравнения с регулярными особыми точками. Для простоты и определенности рассматривается пример вырожденного уравнения Гойна (ВУГ) (о самом уравнении Гойна и различных его вырожденных формах см. [4])

$$
\left(z^{2}+z\right) v^{\prime \prime}+\left(\alpha z^{2}+\beta z+\gamma\right) v^{\prime}+(\delta z+\varepsilon) v=0
$$

где

$$
v^{\prime}(z) \equiv \frac{d v(z)}{d z}
$$

Уравнение (1) имеет две регулярные особые точки $z_{0}=0, z_{1}=-1$ и иррегулярную особую точку на бесконечности. Его частными случаями являются, например, уравнение для кулоновских сфероидальных функций, уравнение для сфероидальных волновых функций [15] и уравнение Матье. Различные вырождения этого 5-параметрического уравнения приводят к ряду других известных дифференциальных уравнений [4].

Опишем вкратце основную идею предлагаемого способа. Рассмотрим вспомогательное уравнение третьего порядка

$$
L_{z}[a, b, c, d, e, f] w(z) \equiv\left(z^{2}+z\right) w^{\prime \prime \prime}+\left(a z^{2}+b z+c\right) w^{\prime \prime}+(d z+e) w^{\prime}+f w=0 .
$$

С одной стороны, оно включает в себя как частный случай ВУГ, а с другой стороны, как будет показано ниже, “устойчиво” относительно интегрального преобразования Эйлера. Уравнение (2) при $f=0$ совпадает с точностью до переобозначений с ВУГ. Этот простой факт позволяет установить соотношение между элементами матриц связи для уравнений (1) и (2). Используя процедуру аналитического продолжения, можно вычислить инвариант преобразования Эйлера в терминах элемента матрицы связи вспомогательного уравнения. Способ вычисления матрицы связи для ВУГ заключается в переходе к пределу по параметру преобразования Эйлера. При этом вспомогательное уравнение преврашается в уравнение с большим параметром, и для вычисления его матрищы связи можно использовать асимптотические методы. 
В отличие от работ $[10,11]$ здесь используются интегральные соотношения, записанные с помошью контурных интегралов. Это позволяет эффективно использовать процедуру аналитического продолжения и вывести необходимые соотношения для элементов матрицы связи.

С формальной точки зрения интегральное преобразование Эйлера для уравнения (2) можно рассматривать как однопараметрическоепреобразование этого уравнения $R(\mu)$, где $\mu$ - свободный параметр преобразования Эйлера, $\mu \in \mathbf{C}$. Нетрудно проверить, что $R\left(\mu_{1}\right) R\left(\mu_{2}\right)=R\left(\mu_{1}+\mu_{2}\right)$. Таким образом, эти преобразования образуют аддитивную однопараметрическую группу. Кроме того, сушествует конечный набор дискретных преобразований (здесь мы их обсуждать не будем).

В следующем разделе введены необходимые понятия и обозначения. В разделе 3 описаны интегральные соотношения для решений уравнения (2). В разделе 4 приведен простой результат, описываюший аналитическое продолжение решения обыкновенного линейного дифференциального уравнения вокруг регулярной особой точки. В пятом разделе приведен результат аналитического продолжения того же решения на основе интегрального соотношения. В разделе 6 получен упомянутый выше инвариант $R(\mu)$. Обсуждается способ вычисления матриц связи для ВУГ на его основе. В этой работе реализация данного способа не проводится. Основной результат статьи - описание однопараметрической группы преобразований Эйлера (точнее, демонстрация того, что уравнение (2) является орбитой этой группы), вычисление ее инварианта (соотношение (21)) в терминах матришы связи уравнения (2) и формулировка способа определения матрицы связи для ВУГ (на основе соотношения (22)).

\section{2. ОСНОВНЫЕ ПОНЯТИЯ}

Напомним известные факты из аналитической теории обыкновенных дифференциальных уравнений $[14,16,17]$ на примере уравнений (1) и $(2)$. Точки $z_{m}(m=0,1)$, являются регулярными особыми точками уравнения (2). В окрестности каждой из этих точек сушествует базис решений уравнения (2), выделенный следуюшим образом. В окрестности $z_{0}$ имеем

$$
W_{01}(z)=z^{2-c} w_{01}(z), \quad W_{02}(z)=z w_{02}(z), \quad W_{03}(z)=w_{03}(z),
$$

а в окрестности $z_{1}-$

$$
W_{11}(z)=(z+1)^{2+a+c-b} w_{11}(z), \quad W_{12}(z)=(z+1) w_{12}(z), \quad W_{13}(z)=w_{13}(z)
$$

причем функции $w_{m k}(z), m=0,1, k=1,2,3$, голоморфны в окрестности точек $z_{m}$, соответственно, и нормированы условиями

$$
w_{m k}\left(z_{m}\right)=1, \quad m=0,1, \quad k=1,2,3 .
$$

Решения, ведущие себя в окрестности особой точки так же, как решения $W_{01}(z)$, $W_{11}(z)$, мы будем в дальнейшем называть "ветвяшимися" в окрестности этой особой 
точки решениями. При этом мы полагаем, что параметры $a, b, c, d, e, f$ уравнения (2) находятся в “обшем положении”, т.е. мы избегаем случайных вырождений типа $c=n$, $n$ целое, когда набор решений уравнения (2) в соответствуюшей особой точке следует надлежащим образом модифицировать.

Введем матрицу связи $T$, связываюшую наборы решений, определенных в окрестности точек $z_{m}$ :

$$
\left(\begin{array}{l}
W_{01}(z) \\
W_{02}(z) \\
W_{03}(z)
\end{array}\right)=\left(\begin{array}{lll}
T_{11} & T_{12} & T_{13} \\
T_{21} & T_{22} & T_{23} \\
T_{31} & T_{32} & T_{33}
\end{array}\right)\left(\begin{array}{l}
W_{11}(z) \\
W_{12}(z) \\
W_{13}(z)
\end{array}\right)
$$

Эта матрища зависит от параметров уравнения (2), так что $T=T(a, b, c, d, e, f)$.

Теорема 1. Матрица $T(a, b, c, d, e, f)$ голоморфно зависит от своих аргуменmos.

Доказательство этого результата, опираюшееся на метод последовательных приближений при построении решений в окрестности регулярных особых точек, мы опускаем.

В дальнейшем будем обсуждать матрицу связи $T$, которая описывает соотношение меж ду наборами решений, фиксированных в окрестности регулярных особых точек. Ситуацию с иррегулярными особыми точками рассмотрим ниже. Основной объект, который нас интересует в дальнейшем, - элемент $T_{11}(a, b, c, d, e, f)$. Покажем, что его величину можно выразить с помощью соответствуюшего элемента матрицы связи для уравнения (1).

Рассмотрим уравнение (1). В окрестности регулярных особых точек можно ввести базис его решений со специальными свойствами:

$$
\begin{array}{ll}
V_{01}(z)=z^{1-\gamma} v_{01}(z), & V_{02}(z)=v_{02}(z), \\
V_{11}(z)=(z+1)^{1+\beta-\alpha-\gamma} v_{11}(z), & V_{12}(z)=v_{12}(z),
\end{array}
$$

причем функции $v_{m k}$ голоморфны в окрестности точек $z_{m}$ и нормированы условиями

$$
v_{m k}\left(z_{m}\right)=1, \quad m=0,1, \quad k=1,2 .
$$

Введем матрищу связи $P$

$$
\left(\begin{array}{l}
V_{01}(z) \\
V_{02}(z)
\end{array}\right)=P\left(\begin{array}{l}
V_{11}(z) \\
V_{12}(z)
\end{array}\right) .
$$

Эта матрица зависит от параметров уравнения (1), так что $P$ есть $(2 \times 2)$-матрица $\left\{P_{i k}(\alpha, \beta, \gamma, \delta, \varepsilon)\right\}, i, k=1,2$. Вообще говоря, матрица связи зависит от пути, вдоль которого осушествляется аналитическое продолжение от одной особой точки к другой. Здесь и далее будем полагать, что этот путь огибает точку $z_{0}$ сверху, начинаясь на вещественной оси правее этой точки и заканчиваясь на вещественной оси правее точки $z_{1}$.

Очевидно, что при $f=0$ уравнение (2) имеет тривиальное решение $W_{m 3}(z) \equiv 1$ и матрица $T$ имеет блочную структуру,

$$
T=\left(\begin{array}{ccc}
T_{11} & T_{12} & 0 \\
T_{21} & T_{22} & 0 \\
0 & 0 & 1
\end{array}\right)
$$


Если при $f=0$ функция $w(z)$ является решением уравнения (2), то функция $w^{\prime}(z)-$ peшение уравнения (1). Диффференшируя верхнюю строку соотношения (5) и сравнивая с верхней строкой соотношения (7), получаем, что

$$
P_{11}(a, b, c, d, e)=\frac{2+a+c-b}{2-c} T_{11}(a, b, c, d, e, 0) .
$$

Здесь учтены условия нормировки (4),(6).

Для определения матришы $P$ достаточно найти только один ее элемент $P_{11}(\alpha, \beta$, $\gamma, \delta, \varepsilon)$, что является следствием элементарных симметрий уравнения (1). Например, рассмотрим преобразование

$$
v(z)=z^{1-\gamma} m(z) .
$$

Нетрудно убедиться, что если $v(z)$ есть решение уравнения (1), то функция $m(z)$ - решение уравнения

$$
\begin{aligned}
& \left(z^{2}+z\right) m^{\prime \prime}+\left[\alpha z^{2}+(\beta+2-2 \gamma) z+2-\gamma\right] m^{\prime}+ \\
& +\left[(\delta+\alpha(1-\gamma)) z+\varepsilon+\beta-\gamma-\beta \gamma+\gamma^{2}\right] m=0
\end{aligned}
$$

которое с точностью до переобозначений совпадает с уравнением (1). Преобразование (8) "переставляет" голоморфное и ветвяшееся в окрестности $z_{0}=0$ решения местами. Учитывая нормировку, получаем, что

$P_{21}(\alpha, \beta, \gamma, \delta, \varepsilon)=\exp (-\pi(1-\gamma) i) P_{11}\left(\alpha, \beta+2-2 \gamma, 2-\gamma, \delta+\alpha(1-\gamma), \varepsilon+\beta-\gamma-\beta \gamma+\gamma^{2}\right)$.

Используя аналогичные соображения для точки $z_{1}$ и симметрию уравнения (1) относительно замены переменной $z=-1-t$, мы можем выразить и остальные элементы матрицы $P$ через $P_{11}$.

Отметим, что если известны матришы связи для данного уравнения, то нетрудно вычислить и матришы монодромии (для любого пути, охватываюшего особенности данного уравнения).

\section{3. ИНТЕГРАЛЬНЫЕ СООТНОШЕНИЯ}

Опишем два интегральных соотношения, которые связывают решения уравнения (2), отвечающие разным значениям параметров уравнения.

Рассмотрим интеграл

$$
w(z)=\int_{C_{m}}(z-t)^{\mu} U_{m 1}(t) d t .
$$

Здесь $U_{m 1}(t)$ - ветвяшееся в окрестности точки $t_{m}\left(m=0,1 ; t_{0}=0, t_{1}=-1\right)$ решение уравнения

$$
L_{t}[a, b+2(\mu+1), c+\mu+1, d+2 a(\mu+1), e+(b+\mu)(\mu+1), f+(d+a \mu)(\mu+1)] u(t)=0,
$$




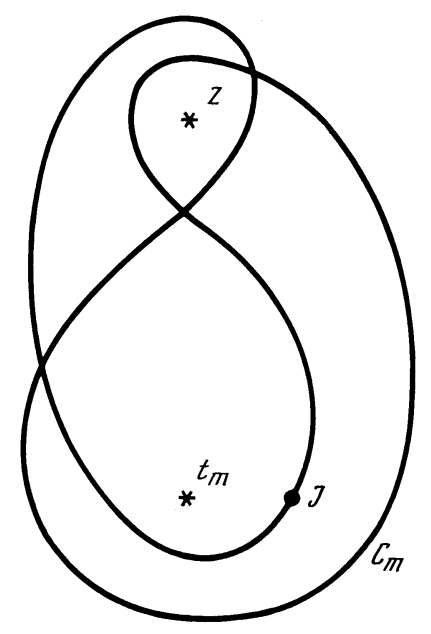

Рис. 1

Это решение нормировано так же, как $W_{m 1}(z)$ из предыдущего раздела. Параметр $\mu \in \mathbf{C}$. Контур интегрирования $C_{m}$ представляет собой двойную восьмерку, охватывающую точки ветвления подынтегральных функций $t=z$ и $t=t_{m}$, соответственно (рис. 1). Фиксируем “начальную” точку контура в точке $J$. В этой точке (находящейся на вещественной оси) фаза подынтегрального выражения полагается вещественной. Мы выбрали такую ветвь контура, после которой точка $t=z$ обходится против часовой стрелки. Данное соглашение (о выборе ветви контура, на которой фиксирована фаза) необходимо для однозначной фиксации нормировок наших функций. Аналогичное соглашение будет использоваться и в дальнейшем. Отметим, что уравнение (10) совпадает с уравнением (2) при $\mu=-1$. Это позволяет отождествлять объекты, относяшиеся к уравнению (2), и соответствующие объекты для уравнения (10) при $\mu=-1$.

Обозначим через $\rho_{m}(\mu)$ показатели ветвления решений $U_{m 1}(t)$. Нетрудно выписать для них явные выражения:

$$
\rho_{1}(\mu)=a+c+1-b-\mu, \quad \rho_{0}(\mu)=1-c-\mu .
$$

ТЕОРЕМА 2. Функция $w(z)$, заданная соотношением (9), с точностью до множителя совпадает с решением $W_{m 1}(z)$ уравнения $(2)$.

ДокАЗАТЕЛЬСТво. Ветвление функции $w(z)$ в окрестности точки $z_{m}$ очевидным образом следует из соотношения (9), ветвления сомножителей в подынтегральном выражении и выбора контура интегрирования. Подставим (9) в (2). С помощью прямых 
вычислений получаем, что

$$
\begin{aligned}
L_{z}[a, b, c, d, e, f] w(z)= & \int_{C_{m}} d t(z-t)^{\mu} u(t)\left\{\frac{z^{2}+z}{(z-t)^{3}} \mu(\mu-1)(\mu-2)+\right. \\
& \left.+\frac{a z^{2}+b z+c}{(z-t)^{2}} \mu(\mu-1)+\frac{d z+e}{z-t} \mu+f\right\}= \\
= & \int_{C_{m}} d t(z-t)^{\mu} u(t)\left\{\frac{t^{2}+t}{(z-t)^{3}} \mu(\mu-1)(\mu-2)+\right. \\
& +\frac{a t^{2}+(b+2 \mu-4) t+c+\mu-2}{(z-t)^{2}} \mu(\mu-1)+ \\
& \left.+\frac{(d+a \mu) t+e+(b+\mu-2)(\mu-1)}{z-t} \mu+f+d \mu+a \mu(\mu-1)\right\} .
\end{aligned}
$$

Интегрируя по частям, приходим к соотношению

$$
\begin{aligned}
L_{z}[a, b, c, d, e, f] w(z)= & \int_{C_{m}} d t(z-t)^{\mu} L_{t}[a, b+2(\mu+1), c+\mu+1, d+ \\
& +2 a(\mu+1), e+(b+\mu)(\mu+1), f+(d+a \mu)(\mu+1)] u(t),
\end{aligned}
$$

что и доказывает теорему.

Вычислим нормировку функции $w(z)$ при $m=0$. В окрестности точки $z_{0}$ имеем

$$
w(z) \sim \int_{C} d t(z-t)^{\mu} t^{1-c-\mu} d t=z^{2-c} \int_{C_{1}} d y(1-y)^{\mu} y^{1-c-\mu}=z^{2-c} Q(c, \mu) .
$$

Здесь использована подстановка $t=z y$. Контур $C$ представляет собой двойную восьмерку на комплексной плоскости, которая охватывает точки $y=0$ и $y=-1$. Аналогичным образом можно вычислить и нормировку при $m=1$. В итоге приходим к следующему результату:

$$
W_{m 1}(z)=\frac{1}{q_{m}(\mu)} \int_{C_{m}}(z-t)^{\mu} U_{m 1}(t) d t
$$

где

$$
q_{m}(\mu)=4 B\left(\rho_{m}(\mu)+1, \mu+1\right) \sin [\pi \mu] \sin \left[\pi \rho_{m}(\mu)\right] \exp \left[-\pi i\left(\rho_{m}(\mu)+\mu\right)\right] .
$$

В качестве контура интегрирования $C_{m}(m=0,1)$ в $(11)$ подразумевается двойная восьмерка, охватываюшая точки $t_{m}, t=z$. Известная эйлерова функция $B(x, y)$ задается либо выражением

$$
B(x, y)=\int_{0}^{1} t^{x-1}(1-t)^{y-1} d t
$$

либо надлежащим аналитическим продолжением.

Приведем еше одно интегральное соотношение. Пусть $\Phi(x)$ - ветвяшееся в окрестности точки $x=0$ решение уравнения

$$
x \Phi^{\prime \prime}(x)+(a x+\nu) \Phi^{\prime}(x)+\rho \Phi(x)=0,
$$


где

$$
\rho=d+a(a-b+\nu) .
$$

Предположим также, что это решение в окрестности точки $x=0$ фиксировано соотношением

$$
\Phi(x) \sim x^{1-\nu} .
$$

Пусть теперь $U_{m 1}(t)$ - ветвяшееся в окрестности $t_{m}(m=0,1)$ и нормированное подобно $W_{m 1}(z)$ решение уравнения

$$
\begin{aligned}
L_{t} & {[a, b+4-2 \nu, c+2-\nu, 2 a(b+1-a-\nu)-d, e-d+} \\
& +2 b-4 \nu+2+(\nu-a)(\nu+a+1-b), f+d(a+\nu+1-b)+ \\
& +2 a(b-a-\nu)+a(a+\nu-b)(a+\nu+1-b)] u(t)=0 .
\end{aligned}
$$

Рассмотрим интеграл

$$
w(z)=\int_{C} \Phi(z-t) U_{m 1}(t) d t,
$$

где $C$ - двойная восьмерка на комплексной плоскости $t$, охватываюшая точки $t=z$ и $t_{m}$.

Теорема 3. Функция $w(z)$, определенная соотношением (16), совпадает с точностью до множителя с решением $W_{m 1}(z)$ уравнения (2).

ДоКАЗАТЕЛЬСТво. То, что функция $w(z)$ ветвится при обходе точки $z_{m}$, следует непосредственно из выбора подынтегральной функции и контура интегрирования. Действуя так же, как при доказательстве теоремы 2 , имеем

$$
\begin{aligned}
L_{z}[a, b, & c, d, e, f] w(z)=\int_{C} d t u(t)\left\{\left(z^{2}+z\right) \Phi^{\prime \prime \prime}+\left(a z^{2}+b z+c\right) \Phi^{\prime \prime}+\right. \\
& \left.+(d z+e) \Phi^{\prime}+f \Phi\right\}=\int_{C} d t u(t)\left\{( z + t + 1 ) \left[(z-t) \Phi^{\prime \prime \prime}+\right.\right. \\
& \left.+(a(z-t)+\nu+1) \Phi^{\prime \prime}+(\rho+a) \Phi^{\prime}\right]+ \\
& +(b-a-\nu-1)\left[(z-t) \Phi^{\prime \prime}+(a(z-t)+\nu) \Phi^{\prime}+\rho \Phi\right]+ \\
& +\left(t^{2}+t\right) \Phi^{\prime \prime \prime}+\left[a t^{2}+(b-2 \nu-2) t+c-\nu-1\right] \Phi^{\prime \prime}+ \\
& +[(2 a(b-a-\nu-1)-d) t+e-d+(\nu-a)(a+\nu+1-b)] \Phi^{\prime}+ \\
& +[f+a(a+\nu-b)(a+\nu+1-b)+d(a+\nu+1-b)] \Phi\} .
\end{aligned}
$$

Для краткости мы не указываем зависимость функции $\Phi$ и ее производных от аргумента $z-t$. Интегрируя по частям и учитьвая уравнение (12), получаем утверждение теоремы 3.

Преобразование, использованное в теореме 2 , известно в литературе как интегральное преобразование Эйлера [14]. По сушеству, теорема 2 показывает “устойчивость" уравнения (2) относительно преобразования Эйлера. Иньми словами, уравнение (2) лежит на орбите этого преобразования. Описанное в теореме 3 интегральное преобразование также зависит от непрерывного параметра. Можно показать, что использованные в теоремах 2 и 3 интегральные преобразования индуцируют на множестве коэффициентов уравнения (2) преобразования, отличаюшиеся лишь дискретным преобразованием. Отметим, что параметр $a$ при этих преобразованиях сохраняется. 


\section{4. АНАЛИТИЧЕСКОЕ ПРОДОЛЖЕНИЕ}

Обсудим на примере уравнения (2) простое соотношение, описывающее результат аналитического продолжения решения линейного дифференциального уравнения вокруг регулярной особой точки.

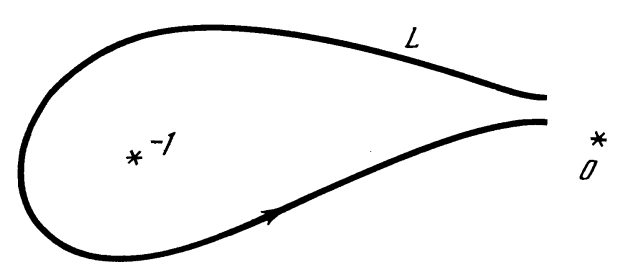

Рис. 2

Рассмотрим поведение решения $W_{01}(z)$ (ветвяшегося при обходе вокруг точки $\left.z_{0}\right)$ при аналитическом продолжении вокруг точки $z_{1}$ вдоль контура $L$ (рис. 2 ). В соответствии с соотношением (5) имеем, что

$$
W_{01}(z)=T_{11} W_{11}(z)+T_{12} W_{12}(z)+T_{13} W_{13}(z) .
$$

Напомним, что $W_{11}(z)$ ветвится при обходе вокруг точки $z_{1}$, а решения $W_{12}(z), W_{13}(z)$ голоморфны в окрестности этой точки. Учитывая соотношение (3), получаем, что в результате аналитического продолжения вдоль контура $L$ функция $W_{01}(z)$ преобразуется согласно соотношению

$$
W_{01}(z) \longmapsto T_{11} \exp [2(2+a+c-b) \pi i] W_{11}(z)+T_{12} W_{12}(z)+T_{13} W_{13}(z) .
$$

Учитывая в последнем соотношении формулу (17), приходим к следуюшему результату:

$$
W_{01}(z) \longmapsto W_{01}(z)+\{\exp [2(2+a+c-b) \pi i]-1\} T_{11} W_{11}(z) .
$$

Соотношение (18), включаюшее в явном виде элемент матрицы связи, позволяет найти инвариант описанных вьше интегральных преобразований, выраженный через этот элемент. Отметим, что аналогичное соотношение справедливо при описании аналитического продолжения вокруг регулярной особой точки любого решения любого линейного обыкновенного дифференциального уравнения.

\section{5. АНАЛИТИЧЕСКОЕ ПРОДОЛЖЕНИЕ С ПОМОЩЬЮ ИНТЕГРАЛА}

В предыдущем разделе мы обсудили результат аналитического продолжения функции $W_{01}(z)$ вдоль контура $L$. Однако есть возможность осушествить аналитическое продолжение вдоль этого контура с помощью интегрального соотношения (11). При $m=0$ имеем

$$
W_{01}(z)=\frac{1}{q_{0}(\mu)} \int_{C_{0}}(z-t)^{\mu} U_{01}(t) d t .
$$




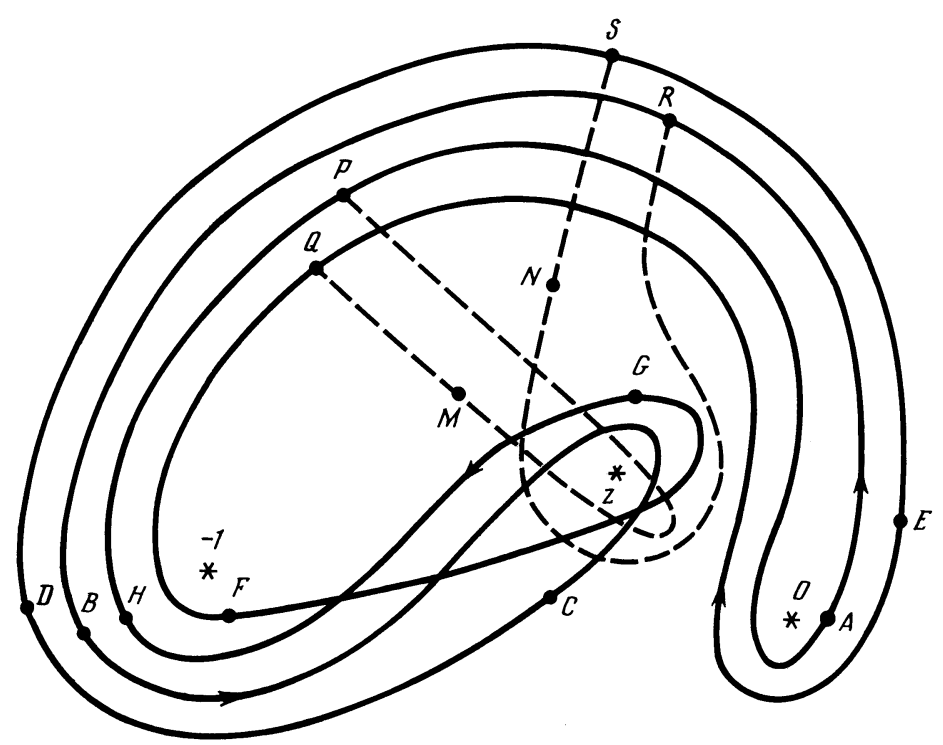

Рис. 3

Пусть аргумент $z$ в этом соотношении движется вдоль контура $L$. В результате получаем

$$
W_{01}(z) \longmapsto \overbrace{W_{01}(z)}=\frac{1}{q_{0}(\mu)} \int_{C_{2}}(z-t)^{\mu} U_{01}(t) d t,
$$

где $C_{2}$ - петля $A R B D E F H A$, результат соответствуюшей деформации исходного контура интегрирования (рис. 3). При этом в подынтегральном выражении следует использовать результат аналитического продолжения функции $U_{01}(t)$ вдоль контура $C_{2}$. Вставим добавочные петли $S N R, P M Q$. Результаты аналитического продолжения подынтегрального выражения (19) вдоль петель $R B D S$ и $R N S$ совпадают. То же справедливо и для петель $Q M P, Q F G H P$. Это означает, что мы можем представить интеграл по контуру $C_{2}$ в виде суммы интегралов по трем контурам:

$$
\int_{C_{2}}=\int_{C_{3}}+\int_{C_{4}}+\int_{C_{5}},
$$

где $C_{3}$ - петля $A R N S E Q M P A, C_{4}$ - петля $B C D S N R B, C_{5}$ - петля $H P M Q F G H$, а в качестве подынтегральных выражений надо понимать результат аналитического продолжения функции $(z-t)^{\mu} U_{01}(t)$ вдоль соответствуюшего контура. При этом подынтегральное выражение вдоль каждого контура является аналитической функцией. Отметим, что контуры $C_{0}$ и $C_{3}$ совпадают, так что интеграл по $C_{3}$ воспроизводит исходное решение.

Рассмотрим контур $C_{6}$ - петлю $H G F Q M P H$, тогда

$$
\int_{C_{5}}=-\int_{C_{6}} .
$$


Для того чтобы описать подынтегральное выражение в интегралах по контурам $C_{4}$ и $C_{6}$, рассмотрим процесс аналитического продолжения подынтегрального выражения. При переходе от точки $t_{0}$ к точке $t_{1}$ вдоль пути, не обходящего вокруг точек $t_{m}$, надо использовать соотношение (аналог верхней строки соотношения (5) при $\mu \neq-1$ )

$$
U_{01}(t)=T_{11}(\mu) U_{11}(t)+T_{12}(\mu) U_{12}(t)+T_{13}(\mu) U_{13}(t) .
$$

Здесь матрица $T(\mu)$ является матришей связи решений уравнения (10), имеющее фиксированное поведение в окрестности точки $t_{0}$, с решениями, фиксированными поведением в окрестности точки $t_{1}$. Если нас интересует аналитическое продолжение подынтегрального выражения вдоль контуров, охватывающих эти точки, необходимо использовать информацию о ветвлении соответствуюшего базиса решений. В результате аналитического продолжения от "начальной" точки контура $C_{0} \mathrm{~K}$ "начальной” точке контура $C_{4}$ получим, что

$$
U_{01} \longmapsto T_{11}(\mu) \exp \left[2 \pi i \rho_{1}(\mu)\right] U_{11}(t)+T_{12}(\mu) U_{12}(t)+T_{13}(\mu) U_{13}(t) .
$$

Заметим, что решения $U_{12}(t)$ и $U_{13}(t)$ голоморфны в окрестности точки $t_{1}=-1$, так что при интегрировании по контурам $C_{4}, C_{6}$ их вклады пропадут. Таким образом имеем

$$
\begin{aligned}
& \int_{C_{4}}(z-t)^{\mu} U_{01}(t) d t=T_{11}(\mu) \exp \left[2 \pi i \rho_{1}(\mu)\right] \int_{C_{4}}(z-t)^{\mu} U_{11}(t) d t, \\
& \int_{C_{6}}(z-t)^{\mu} U_{01}(t) d t=T_{11}(\mu) \exp \left[2 \pi i \rho_{1}(\mu)-2 \pi i \rho_{0}(\mu)\right] \int_{C_{6}}(z-t)^{\mu} U_{11}(t) d t .
\end{aligned}
$$

Контуры $C_{4}, C_{6}$ с точностью до очевидной деформации совпадают с контуром $C_{1}$. Следовательно, соответствуюшие интегралы могут быть вычислены с помощью соотношения (11). В итоге находим, что

$$
W_{01}(z) \longmapsto W_{01}(z)+\frac{q_{1}(\mu)}{q_{0}(\mu)} T_{11}(\mu) \exp \left[2 \pi i \rho_{1}(\mu)\right]\left\{1-\exp \left[-2 \pi i \rho_{0}(\mu)\right]\right\} W_{11}(z) .
$$

\section{6. ИНТЕГРАЛЬНЫЙ ИНВАРИАНТ И СПОСОБ ВЫЧИСЛЕНИЯ МАТРИЦЫ СВЯЗИ ДЛЯ ВУГ}

Мы осуществили аналитическое продолжение одной и той же функции вдоль одного и того же контура двумя разными способами. Сравнивая соотношение (20) с формулой (18), получаем

$$
\{\exp [2(2+a+c-b) \pi i]-1\} T_{11}=\frac{q_{1}(\mu)}{q_{0}(\mu)} \exp \left[2 \pi i \rho_{1}(\mu)\right]\left\{1-\exp \left[-2 \pi i \rho_{0}(\mu)\right]\right\} T_{11}(\mu) .
$$

Это соотношение и является искомым инвариантом интегрального преобразования. В самом деле, в левой его части стоит выражение, не зависяшее от $\mu$. Таким образом, и правая его часть от этого параметра не зависит. Отметим, что в случае предельного перехода $\mu \rightarrow-1$ правая часть (21) совпадает с левой (в силу теоремы 1 имеем, что 
$\left.T_{11}(\mu) \rightarrow T_{11}(-1)\right)$. Соотношение (21) описывает преобразование решений при переходе от одной регулярной особой точки к другой. Аналогичные методы позволяют найти подобное соотношение и для матриц связи, описываюших переход от регулярной особой точки к иррегулярной.

В частности, соотношение (21) сохраняется и тогда, когда параметр $\mu$ стремится к бесконечности. Это дает возможность получить следуюшее следствие из соотношения (21):

$$
\begin{aligned}
& \{\exp [2(2+a+c-b) \pi i]-1\} T_{11}(-1)= \\
& \quad=\lim _{\mu \rightarrow i \infty}\left\{\frac{q_{1}(\mu)}{q_{0}(\mu)} \exp \left[2 \pi i \rho_{1}(\mu)\right]\left\{1-\exp \left[-2 \pi i \rho_{0}(\mu)\right]\right\} T_{11}(\mu)\right\} .
\end{aligned}
$$

Из соотношения (22) следует, что для определения элемента матрицы связи $T_{11}(-1)$ достаточно вычислить аналогичный элемент матришы связи для подобного же уравнения, обладающего большим параметром. Таким образом, аналитическая задача вычисления $T_{11}(-1)$ сведена к задаче асимптотического анализа. Точка $i \infty$ выбрана с целью исключить осложнения типа экспоненциально больших или экспоненциально малых вкладов при асимптотических вычислениях.

На основе описанных результатов можно предложить способ вычисления элемента матрицы связи $T_{11}(-1)$ (полагая при этом $f=0$ ), а затем, как отмечено выше, и всей матрицы связи для ВУГ. Необходимо построить асимптотические (по большому параметру) решения уравнения (10) с фиксированным поведением в окрестности точек $t_{0}$ и $t_{1}$. Вдали (т.е. на расстояниях порядка $O(1)$ ) от этих точек мы должны сравнить эти решения и выразить один набор решений через другой. Определив элемент $T_{11}(\mu)$, следует подставить его в правую часть выражения (21) и перейти к пределу $\mu \rightarrow i \infty$. При построении решений уравнения (10) с надлежашим поведением при $\mu \rightarrow i \infty$ можно использовать соответствуюшую модификацию метода эталонного уравнения. Разумеется, это весьма громоздкая задача. Однако ожидаемые при ее реализации трудности носят скорее технический характер.

\section{7. ЗАКЛЮЧЕНИЕ}

В предыдушем разделе описан способ вычисления матрицы связи для ВУГ. Эта аналитическая проблема сведена к некоторой асимптотической задаче. Разумеется, последняя требует для своего решения достаточно громоздких вычислений. Однако опыт асимптотических вычислений позволяет надеяться на возможность ее решения.

Отметим еще раз основные моменты предлагаемой схемы нахождения матрицы связи для уравнения с регулярными особыми точками. Мы рассматриваем действие на множестве линейных дифференциальных уравнений интегрального преобразования типа (16), взяв в качестве функции $\Phi(x)$ ветвяшуюся в окрестности точки $x=0$ функцию. Эта функция может быть решением обыкновенного линейного дифференциального уравнения со свободными параметрами и регулярной особой точкой при $x=0$ (самый простой вариант, ядро Эйлера, описан в теореме 2 , другой способ указан в теореме 3). У рассматриваемого интегрального преобразования есть орбиты, т.е. семейства 
линейных дифференциальных уравнений. Среди этих орбит следует найти ту, на которой находится исходное уравнение. Для ВУГ это уравнение (2) (гипергеометрическое уравнение "устойчиво" под действием преобразования Эйлера [1]). После этого с помошью аналитического продолжения можно получить инвариант этих интегральных преобразований, выраженный через соответствуюшие матрицы связи. Данный результат позволяет предложить способ вычисления матрицы связи с помошью подходяшего предельного перехода на основе асимптотической техники.

Мы обсуждали случай матрицы связи, соответствуюшей паре регулярных особых точек. В окрестности иррегулярной особой точки более сложными являются построение самого эталонного уравнения и реализация метода эталонного уравнения.

Автор благодарен В. С. Булдыреву, И. В. Комарову и С. Ю. Славянову за интерес к работе и ценную дискуссию.

Работа поддержана грантом РФФИ 98-01-01075.

\section{Список литературы}

[1] Г. Бейтмен, А. Эрдейи. Высшие трансцендентные функции. Т. 1. М.: Наука, 1965.

[2] И. С. Градштейн, И. М. Рыжик. Таблицы интегралов, сумм, рядов и произведений. М.: ГИФМЛ, 1962.

[3] M. Abramovitz, I.A. Stegun. Handbook of mathematical functions. Dover Inc., 1968.

[4] Centennial Workshop on Heun's equation. Eds A. Seeger, W. Lay. Stuttgart: Max-Plank-Institut für Metallforschung, 1990.

[5] A. Erdelyi. Quart. J. Math. 1942. V. 13. P. 107.

[6] Д. И. Абрамов, И. В. Комаров. ТМФ. 1976. Т. 29. С. 235.

[7] B.C. Carlson. SIAM J. Math. Anal. 1980. V. 11. P. 325.

[8] G. Valent. SIAM J. Math. Anal. 1986. V. 17. P. 688.

[9] А.Я. Казаков. Дифф. уравнения. 1986. Т. 22. С. 354.

[10] A. Ya.Kazakov, S. Yu.Slavyanov. Meth. Appl. Anal. 1996. V. 3. P. 447.

[11] А.Я. Казаков, С. Ю. Славянов. ТМФ. 1996. Т. 107. С. 388.

[12] W. Buhring. A linear differential equation with two irregular singular points of rank one. In: Centennial Workshop on Heun's equation. Eds A. Seeger, W. Lay. Stuttgart: Max-Plank-Institute für Metallforschung, 1990.

[13] A. А. Болибрух. 21 проблема Гильберта для линейных фуксовых систем. Тр. МИРАН. 1994. T. 206.

[14] E. Hille. Ordinary differential equations in the complex domain. New York: Dover Publications, Inc. Mineola, 1997

[15] И. В. Комаров, Л. И. Пономарев, С. Ю. Славянов. Сфероидальные и кулоновские сфероидальные функции. М.: Наука, 1976.

[16] Ф. Трикоми. Дифференциальные уравнения. М.: ИИЛ, 1962.

[17] В. В. Голубев. Лекции по аналитической теории дифференциальных уравнений. М.-Л.: Гостехиздат, 1941. 